\author{
MITSUBISHI ELECTRIC RESEARCH LABORATORIES \\ http://www.merl.com
}

\title{
Interference Management with Han-Kobayashi Coding: Dual-Carrier Coherent Optical Communications
}

\author{
Koike-Akino, T.; Kojima, K.; Millar, D.S.; Parsons, K.; Kametani, S.; Sugihara, T.; Yoshida, T.; \\ Ishida, K.; Miyata, Y.; Matsumoto, W.; Mizuochi, T.
}

TR2014-060 September 2014

\begin{abstract}
We propose to use Han-Kobayashi (HK) coding and dirty-paper coding (DPC) to cope with inter-carrier interference (ICI) in dual-carrier transmissions. We show the considerable benefit of those methods to increase throughput in presence of strong ICI for dense carrier spacing.
\end{abstract}

European Conference on Optical Communications (ECOC 2014)

This work may not be copied or reproduced in whole or in part for any commercial purpose. Permission to copy in whole or in part without payment of fee is granted for nonprofit educational and research purposes provided that all such whole or partial copies include the following: a notice that such copying is by permission of Mitsubishi Electric Research Laboratories, Inc.; an acknowledgment of the authors and individual contributions to the work; and all applicable portions of the copyright notice. Copying, reproduction, or republishing for any other purpose shall require a license with payment of fee to Mitsubishi Electric Research Laboratories, Inc. All rights reserved.

Copyright (C) Mitsubishi Electric Research Laboratories, Inc., 2014

201 Broadway, Cambridge, Massachusetts 02139 



\title{
Interference Management with Han-Kobayashi Coding: Dual-Carrier Coherent Optical Communications
}

\author{
Toshiaki Koike-Akino(1), Keisuke Kojima(1), David S. Millar ${ }^{(1)}$, Kieran Parsons ${ }^{(1)}$, \\ Soichiro Kametani(2), Takashi Sugihara(2), Tsuyoshi Yoshida(2), Kazuyuki Ishida ${ }^{(3)}$, \\ Yoshikuni Miyata $^{(2)}$, Wataru Matsumoto ${ }^{(2)}$, Takashi Mizuochi(4) \\ (1) Mitsubishi Electric Research Labs., 201 Broadway, Cambridge, MA 02139, USA, koike@merl.com \\ (2) Information Technology R\&D Center, Mitsubishi Electric Corp., 5-1-1, Ofuna, Kamakura, Japan \\ (3) Communication Networks Center, Mitsubishi Electric Corp., 8-1-1, Tsukaguchi, Amagasaki, Japan \\ (4) Advanced Technology R\&D Center, Mitsubishi Electric Corp., 8-1-1, Tsukaguchi, Amagasaki, Japan
}

\begin{abstract}
We propose to use Han-Kobayashi (HK) coding and dirty-paper coding (DPC) to cope with inter-carrier interference $(\mathrm{ICl})$ in dual-carrier transmissions. We show the considerable benefit of those methods to increase throughput in presence of strong ICI for dense carrier spacing.
\end{abstract}

\section{Introduction}

Rapid demand of the data-rate increase has necessitated high-throughput technologies, such as faster-than Nyquist ${ }^{1,2}$ and super-channel transmissions ${ }^{3-5}$, where each transceiver operates independently while increasing the total throughput without increasing the processing speed. The spectrum efficiency may increase as the carrier spacing decreases. However, intercarrier interference $(\mathrm{ICl})$ can be a major limiting factor to realize a dense carrier spacing, and $\mathrm{ICl}$ is often desired to be minimized.

For overlapped dual-carrier transmissions, we can model the system as a 2-user interference channel. Han and Kobayashi have shown ${ }^{6}$ that a very strong interference cannot limit the channel capacity because interference conveys data sent from the adjacent channel and those message can be decoded for strong interference cases. This theory suggests that suppressing undesired interference may not always be the best solution. Even for weak interference cases, the best known rate is achieved by the Han-Kobayashi (HK) scheme $e^{6-8}$, which uses a super-position coding and a successive decoding.

In this paper, we study a potential benefit obtained by the HK scheme for dual-carrier transmissions. In addition, we compare the achievable rate of joint decoding and dirty-paper coding $(\mathrm{DPC})^{9,10}$. The major contributions are twofold: i) we analyze the benefit of HK scheme for different filter parameters, and ii) we investigate the impact of equalizer memory length in dual-carrier transmissions. To the best of authors' knowledge, there has been no literature which applied HK or DPC to optical communications.

\section{Interference in dual-carrier transmissions}

Fig. 1 depicts a schematic of dual-carrier coherent optical transmissions, employing the

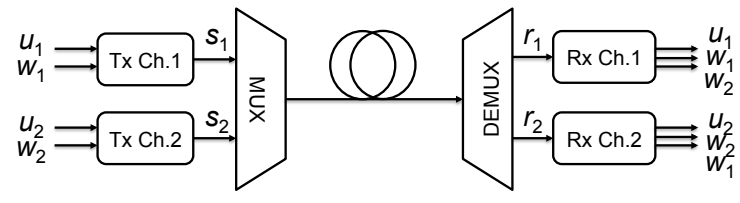

Fig. 1: Dual-carrier transmission systems with HK scheme.

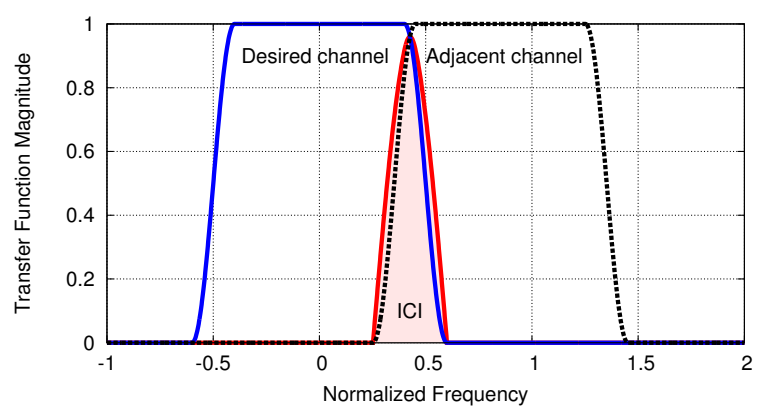

Fig. 2: Transfer functions in overlapped dual-carrier transmissions.

HK scheme. Two transmitters send codedmodulation messages $s_{1}$ and $s_{2}$, respectively, at carrier frequencies $f_{1}$ and $f_{2}$. Letting $B$ be the baud rate, the carrier spacing normalized by the baud rate is written as $\delta f=\left(f_{2}-f_{1}\right) / B$. For simplicity, we assume root raised-cosine (RRC) filters with a roll-off factor of $\alpha$ for both Tx and $\mathrm{Rx}$ filters. We consider the case when the carrier spacing is dense such that $\delta f<1+\alpha$, leading to $\mathrm{ICl}$ as shown in Fig. 2, where $\delta f=0.85$ and $\alpha=0.2$.

For a carrier $f_{k}(k \in\{1,2\})$, the receiver obtains $r_{k}$, passing through optical fibers and TRx filters. This system can be 'conceptually' modeled as

$$
\begin{aligned}
& r_{1}=s_{1}+\sqrt{\beta} s_{2}+n_{1}, \\
& r_{2}=\sqrt{\beta} s_{1}+s_{2}+n_{2},
\end{aligned}
$$

where $\beta$ is a ratio between interference and desired signal powers, and $n_{k}$ is an additive Gaussian noise with a variance of $1 / \rho$ for a 
signal-to-noise power ratio (SNR) of $\rho$. Since in reality the $\mathrm{ICl}$ signal has memory and phase rotation, this simplified model will be modified and discussed in more detail later.

\section{Han-Kobayashi coding and decoding}

The HK scheme ${ }^{6-8}$ uses super-position coding and partial joint decoding. For transmitters, the coded sequence $s_{k}$ is a super-position of two codewords of source messages $u_{k}$ and $w_{k}$. One is for private messages, which are decoded only at the intended receiver. The other is used for public messages, which are decoded at receivers for both carriers. Two codewords (for private $u_{k}$ and public messages $w_{k}$ ) are superposed with a power splitting of $\lambda_{k}$ and $1-\lambda_{k}$.

The achievable data rate of HK scheme for a symmetric power splitting case $\lambda_{1}=\lambda_{2}$ is given ${ }^{8}$ by

$R_{\mathrm{HK}}= \begin{cases}2 C\left(\frac{\rho}{1+\beta \rho}\right), & \rho \leq \rho_{1}, \\ 2 C\left(\frac{\left(\beta^{2} \rho+\beta-1\right)(1-\beta)+\beta \rho}{1+\beta\left(\beta^{2} \rho+\beta-1\right)}\right), & \rho_{1}<\rho \leq \rho_{2}, \\ C\left(\frac{1-\beta}{2 \beta}\right)+C\left(\frac{(1+\beta)^{2} \rho-(1-\beta)}{2}\right), & \rho_{2}<\rho,\end{cases}$ where $\rho_{1}=\frac{1-\beta}{\beta^{2}}, \rho_{2}=\frac{1-\beta^{3}}{\beta^{3}(\beta+1)}$, and $C(\rho)=\log _{2}(1+$ $\rho)$. When allowing an asymmetric power splitting with $\lambda_{1}=0$ and $\lambda_{2}>0$, slightly higher data rates in high SNR regimes can be achieved ${ }^{8}$.

For the case when all messages are private with $\lambda_{1}=\lambda_{2}=1$ (corresponding to a conventional coding method), the achievable rate reduces to

$$
R_{\text {conv }}=2 C\left(\frac{\rho}{1+\beta \rho}\right) .
$$

On the contrary, for the case when the all messages are public with $\lambda_{1}=\lambda_{2}=0$ (i.e., joint decoding scheme), the achievable rate becomes

$$
R_{\text {joint }}= \begin{cases}2 C\left(\frac{\rho}{1+\beta \rho}\right), & \rho \leq \rho_{1}, \\ C((1+\beta) \rho), & \rho_{1}<\rho .\end{cases}
$$

When one transmitter knows the message of the other transmitter, we can use the DPC scheme $^{9}$ to cancel the interference in advance like Tomlinson-Harashima precoding, achieving the rate:

$$
R_{\mathrm{DPC}}=C(\rho)+C\left(\frac{\rho}{1+\beta \rho}\right) .
$$

One transmitter requires twice-times higher processing speed to use DPC. Note that a practical DPC based on repeat-accumulate codes is reported by Erez and ten Brink ${ }^{10}$.

\section{Memory of inter-channel interference}

The model of $\mathrm{ICI}$ should be modified by taking the memory of the interference impulse response into account, as shown in Fig. 3, where the impulse response magnitude of the $\mathrm{ICl}$ component is plotted for $\alpha \in\{0.2,0.05\}$ and $\delta f=0.85$. It is seen that the impulse response of the desired signal has no inter-symbol interference (ISI) at a sample of symbol timing because of the Nyquist criterion. Although the peak power of the impulse response for $\mathrm{ICl}$ is lower than the desired signal, $\mathrm{ICI}$ has longer memory especially for a smaller roll-off factor.

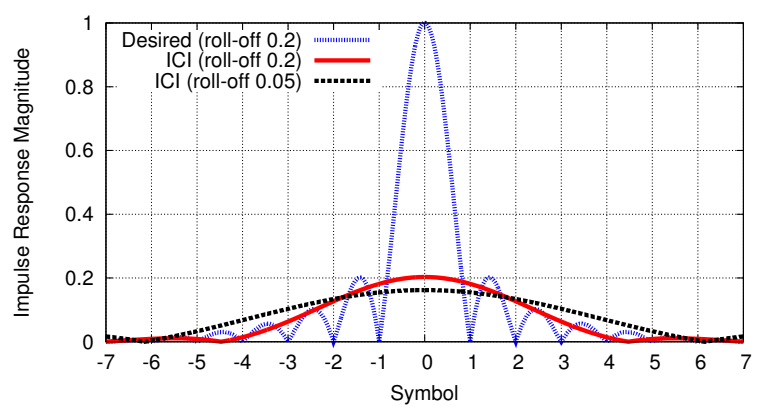

Fig. 3: Impulse response magnitude of desired and interference signals $(\alpha=\{0.2,0.05\}, \delta f=0.85)$.

Therefore, we need an equalizer to decode interference signals for HK, DPC and joint decoding. Let $L$ and $h(z)$ be the equalizer memory length and the discrete-time complex-valued impulse response of $\mathrm{ICl}$ signal, respectively. Using a finite-memory maximum a posteriori probability (MAP) equalization, the signal power within the memory $\sum_{k=-L / 2}^{L / 2}|h(k)|^{2}$ contributes to the interference power in $\beta$, and the reminder outside of the memory adds up the noise variance $1 / \rho$.

\section{Performance analysis}

We evaluate the performance of HK and DPC in dual-carrier transmissions. To obtain spectrum efficiency, the achievable sum rates $\left(R_{\mathrm{HK}}, R_{\text {conv }}\right.$, $R_{\text {joint }}$, and $\left.R_{\text {DPC }}\right)$ are normalized by a total bandwidth consumption of $B(1+\alpha+\delta f)$.

Fig. 4 shows the throughput as a function of roll-off factor $\alpha$ for 3-tap and 19-tap equalizers at a noise spectrum density of $-30 \mathrm{dBc}$ and a carrier spacing of $\delta f=0.85$. We can see that a conventional scheme (which does not consider ICI) degrades with the increased roll-off factor because of the increasing noise and interference power. For 3-tap equalizers, the throughput can be increased with HK, DPC and joint decoding particularly at around $\alpha=0.6$. For 19-tap longmemory equalizers, HK and DPC provide a significant gain at around $\alpha=0.2$. It should be noted that minimizing $\mathrm{ICl}$ with $\alpha=0$ is not optimal for those schemes.

At a roll-off factor of $\alpha=0.2$, the performance curves against the carrier spacing $\delta f$ are plotted 


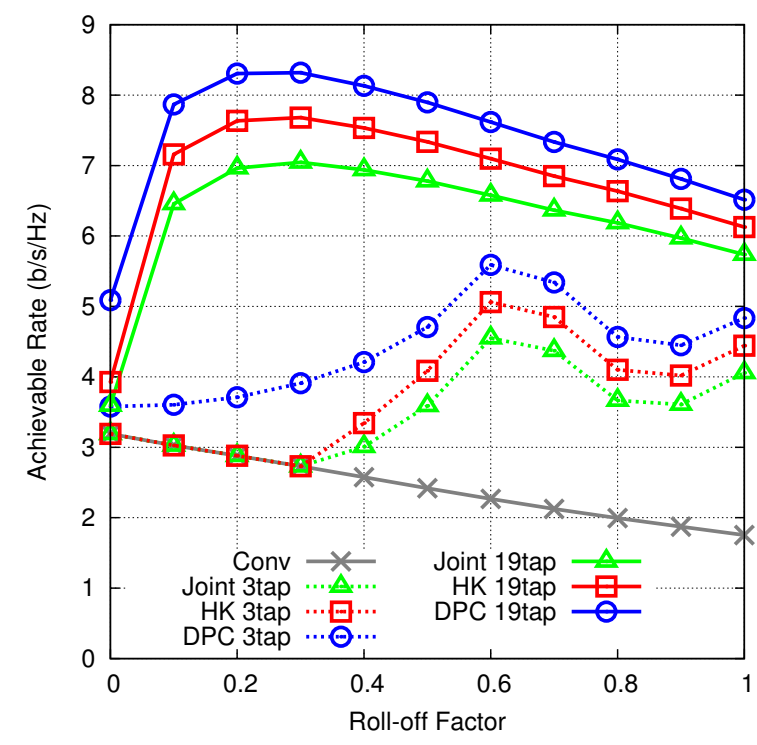

Fig. 4: Achievable rate vs. roll-off factor $\alpha(\delta f=0.85)$.

in Fig. 5. For low ICI regimes such that $\delta f>1$, there is few gain of the HK scheme. Whereas, the HK, DPC and joint decoding can achieve $2 \sim 5$ times higher throughput than conventional scheme in strong ISI regimes when long-memory equalizers are used.

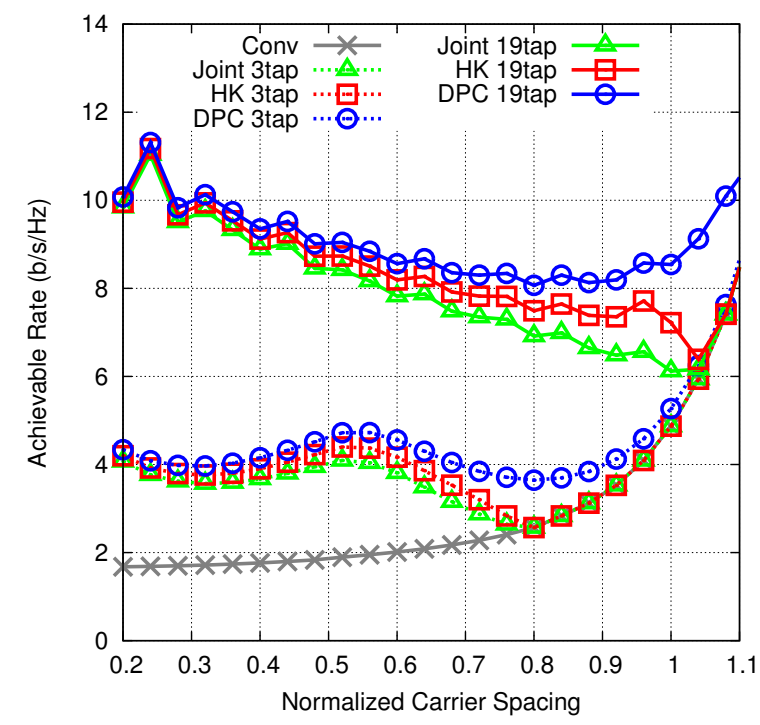

Fig. 5: Achievable rate vs. carrier spacing $\delta f(\alpha=0.2)$.

The impact of the equalizer memory is shown in Fig. 6 . It is demonstrated that the longer memory offers better performance with $\mathrm{HK}$, and that less than 5-tap memory cannot outperform the conventional way. It is because the $\mathrm{ICl}$ signal cannot be decoded with shorter-memory equalization than the memory of ISI impulse response especially for small roll-off factor cases.

\section{Conclusions}

We have shown a significant benefit of HK scheme to deal with $\mathrm{ICI}$ in dual-carrier transmissions. Compared to a conventional scheme,

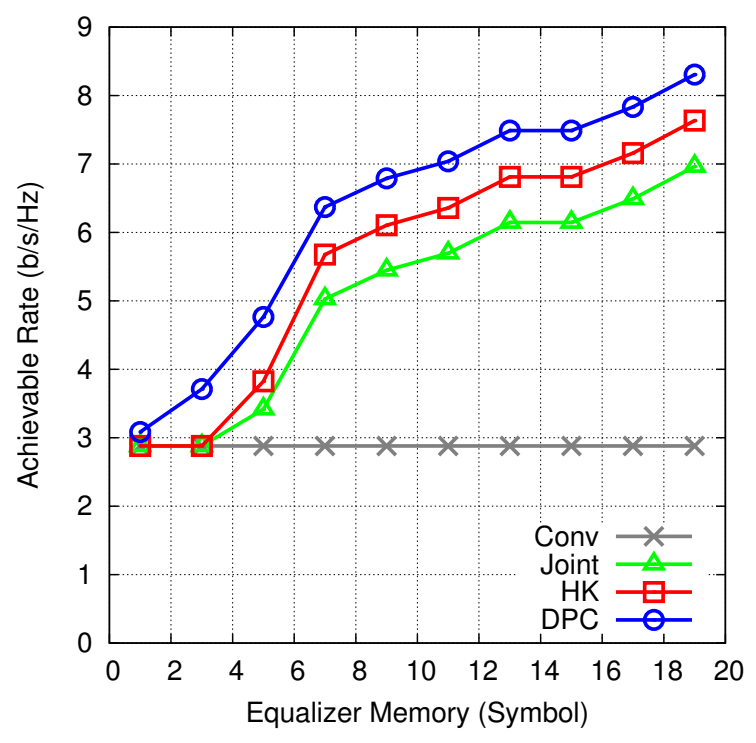

Fig. 6: Achievable rate vs. equalizer memory $(\alpha=0.2$, $\delta f=0.85)$.

HK scheme achieves approximately twice higher throughput for the case of overlapped carrier spacing with long-memory equalizers. We have also compared with DPC and joint decoding, respectively showing a slightly better and worse performance than HK scheme. The generalization to multiple carriers and detail evaluation in nonlinear fiber channels remain as a future work. With nonlinearity, HK may be more useful because $\mathrm{ICl}$ can increase.

\section{Acknowledgments}

This work was in part supported by the Lambda Reach project of the National Institute of Information and Communications Technology (NICT), as part of a program of the Ministry of Internal Affairs and Communications (MIC) of Japan.

\section{References}

[1] E. Mazo, Bell System Tech. J. 54 (1975) 14501462.

[2] G. Colavolpe, T. Foggi, A. Modenini, and A. Piemontese, Opt. Express 19 (2011).

[3] B.-E. Olsson, A. Kristiansson, and A. Alping, OFC, OM.K5 (2010).

[4] V. Sleiffer, et al., OFC OW4C-3 (2012).

[5] H.-S. Chung, OFC JW2A-3 (2012).

[6] T. Han and K. Kobayashi, IEEE Trans. IT 27, no. 1, pp. 49-60, Jan. 1981.

[7] R. Etkin, D. Tse, and H. Wang, IEEE Trans. IT 54, no. 12, pp. 5534-5562, Dec. 2008.

[8] O. Mehanna, J. Marcos, and N. Jindal, Allerton Conf. on Communication, Control, and Computing, IL, Sept. 2010.

[9] M. Costa, IEEE Trans. IT 29, pp. 439-441, May 1983.

[10] U. Erez and S. ten Brink. IEEE Trans. IT 51, no. 10 (2005) 3417-3432. 